\section{Qualitätsstandards für die Stationäre Psychiatrie}

\section{H. Wengle}

Art. 58 KVG sowie Art. 77 Abs. 1 KVV verpflichten die Spitäler und Versicherer, Qualitätsverträge abzuschliessen. Die Kliniken sind angehalten, ihre Prozesse zu optimieren, eigene Stärken zu pflegen und Schwächen entgegenzuwirken. Die Arbeit an der Qualität hat eine starke situative und lokale Komponente. Jede Klinik muss ihren eigenen Weg zu mehr Qualität finden. Dabei kann sie von der Erfahrung anderer Kliniken lernen. Die Präsidenten der Schweizerischen Vereinigung Psychiatrischer Chefärzte SVPC, der Konferenz der Pflegedienstleitungen Psychiatrischer Institutionen KPP und der Arbeitsgemeinschaft der Verwaltungsleiter Psychiatrischer Kliniken der Schweiz AVPS haben deshalb die Psychiatrischen Kliniken zur Zusammenarbeit aufgerufen.

Im Januar 1999 hat sich nach einiger Vorarbeit unter dem Dach der Schweizerischen Vereinigung Psychiatrischer Chefärzte SVPC eine interdisziplinäre Arbeitsgruppe ${ }^{1}$ konstituiert. 22 Psychiatrische Kliniken in der Deutschschweiz haben der SVPC bzw. der Arbeitsgruppe daraufhin den Auftrag erteilt, Qualitätsstandards auszuarbeiten. Ein erster Entwurf wurde bei 50 Kliniken und Experten in Vernehmlassung gegeben. Dadurch sollte sichergestellt werden, dass die Themen relevant, die Standards konsensfähig und den Bedürfnissen der Kliniken entsprechen. Aufgrund der Rückmeldungen wurden einige Themen verworfen, neue aufgenommen und bestehende revidiert. Im August sind neun Standards für die Stationäre Psychiatrie gedruckt worden. ${ }^{2}$ Eine französische und eine italienische Fassung werden folgen.

1 Hanspeter Wengle, Chefarzt, Wil; Rolf Bitterlin, Leiter Pflegedienst, Oetwil am See; Hans Joachim Haug, Chefarzt, Zürich; Jürg Schaefer, Verwaltungsdirektor, Embrach.

2 Zu beziehen bei Dr. med. U. Fromm, Präsident SVPC, Externer Psychiatrischer Dienst des Kantons Aargau, Westallee 2, 5000 Aarau; nähere Angaben auch unter www.psychiatrie.ch.

Korrespondenz:

Dr. med. Hanspeter Wengle

Kantonale psychiatrische Klinik

Zürcherstrasse 30

CH-9500 Wil SG
Folgende Standards wurden vorgelegt:

Behandlungsstandards (B-Standards)

B1 Drei kritische Abläufe in der Behandlung: Aufnahme, Behandlungsvereinbarung, Austritt.

B2 Umgang mit Risikosituationen.

B3 Zwangsmassnahmen: Isolation, Fixation, Zwangsinjektion.

B4 Behandlung auf wissenschaftlicher Grundlage. B5 Patientenzufriedenheit.

\section{Die Behandlung unterstützende Standards}

(U-Standards)

U1 Interdisziplinäre Zusammenarbeit.

U2 Umgang mit Patientendaten.

U3 Das Mitarbeitergespräch.

U4 Integriertes medizinisch-ökonomisches Denken und Handeln.

Die Arbeitsgruppe hat einen pragmatischen Ansatz verfolgt und die Standards so konzipiert, dass sie vor allem für jene Kliniken geeignet sind, die einen praxis- und projektorientierten Einstieg suchen. Die Standards sind thematisch nicht "flächendeckend". Die einzelnen Projekte müssen zu einem späteren Zeitpunkt in ein Qualitätsmanagementsystem eingebaut werden. Die Standards legen einen Schwerpunkt auf Prozessorientierung, Nahtstellenprobleme und kontinuierliche Arbeit an der Qualität. Sie sind so formuliert, dass die Kliniken Freiräume haben und ihre eigene, gewachsene Praxis weiterführen und -entwickeln können und müssen. Ein Begleittext führt den Kontext der Standards weiter aus. Es werden Hinweise gegeben, wie mit den Standards gearbeitet und das erreichte Qualitätsniveau beurteilt werden kann. Die Arbeit mit Standards bringt in jedem Fall viel Aufwand mit sich. Sie können zwar Wissen vermitteln, anregen, unterstützen, vereinfachen und Irrwege ersparen, den Kliniken aber die Arbeit nicht abnehmen.

Das Besondere der Standards ist, dass sie die Behandlung selbst thematisieren. Die fachlichen Vorgaben einer Behandlung werden durch Guidelines vermittelt. Die vorgelegten Standards unterstützen die optimale Ausgestaltung der Behandlungsprozesse inkl. Risikominimierung. Die Standards sind qualitäts-, methoden- und strategieneutral. Als Arbeitsinstrumente helfen sie, relevante Qualitätsprojekte aufzubauen und allenfalls externe Qualitätsforderungen unter dem Rahmenvertrag $\mathrm{H}+/ \mathrm{KSK}$, unter EFQM, ISO u.a. zu erfüllen.

Die genannten Vereinigungen SVPC, KPP und AVPS werden im März 2001 die Leitungen der Psychiatrischen Kliniken zu einer Tagung einladen. Die Veranstaltung soll einen Überblick über den Stand der Qualitätsbemühungen der Kliniken vermitteln und über die vorgelegten Standards informieren. SVPC, KPP und AVPS leisten mit dieser Tagung Geburtshilfe. Es wird dann an den interessierten Kliniken liegen, sich zu organisieren. In diesem Sinne sind die Standards als Hilfe zur Selbsthilfe für interessierte Psychiatrische Kliniken zu verstehen. 
Im folgenden werden die Standards ausführlicher dargestellt:

\section{B1 Drei kritische Abläufe in der Behandlung: Aufnahme, Behandlungsvereinbarung, Austritt}

Der Standard konzentriert sich auf drei besonders kritische Elemente im Verlauf einer stationären Behandlung: Auf die Aufnahme, die Behandlungsvereinbarung und den Austritt. Es handelt sich dabei um Kernelemente der Behandlung, die nicht nur je für sich, sondern vor allem in ihrem Ineinandergreifen und Zusammenwirken qualitätsrelevant sind. Deshalb werden die drei Elemente in einem Standard zusammengefasst. Die Herausforderung für die Klinik liegt unter anderem darin, dass nicht nur die medizinisch-fachlichen, sondern auch die organisatorischen und vor allem die psychologischen Aspekte der drei Elemente optimiert und aufeinander abgestimmt werden müssen.

\section{B2 Umgang mit Risikosituationen}

Während jeder stationären Behandlung können sich Risikosituationen einstellen, bei denen Patienten oder die Klinik potentiell oder tatsächlich Schaden nehmen. Für den Patienten kann dies ein materieller, ein körperlicher oder psychischer Integritätsschaden sein. Die Klinik sieht sich dann möglicherweise mit öffentlicher Kritik, mit finanziellen Forderungen und/oder juristischen Verfahren konfrontiert und kann ihrerseits einen materiellen oder immateriellen Schaden erleiden. Der Standard beschränkt sich auf jene Risikosituationen, die der Klinik Anlass zu besonderen Massnahmen geben, d.h. zu Massnahmen, die über die Behandlungsroutine hinausgehen. Risiken im Sinne dieses Standards sind beispielsweise Suizidalität/Suizid, Fremdgefährdung/Tätlichkeiten, Stürze, Medikamentenfehler, Unfälle und Berufskrankheiten, sexuelle Übergriffe, Diebstähle, aber auch Reklamationen, Beschwerden, Klagen, finanzielle Forderungen usw.

\section{B3 Zwangsmassnahmen:}

\section{Isolation, Fixation, Zwangsinjektion}

Die meisten Psychiatrischen Kliniken haben eine Aufnahmepflicht. Sie müssen Patienten auch gegen ihren Willen aufnehmen und behandeln. Dieser gesellschaftliche Auftrag wird durch gesetzliche Bestimmungen legitimiert, die gleichzeitig die Rechte der Patienten schützen. Zwang kann notwendig sein, um für Patienten und Mitarbeitende ein Milieu herzustellen, das frei von physischer Bedrohung ist. Ein sicheres Milieu ist für den Heilungsprozess notwendig und förderlich. Der Auftrag, Zwangsmassnahmen durchführen zu müssen, hat komplexe Auswirkungen auf die psychiatrischen Kliniken. Will die Klinik Zwang nur als letztes Mittel und so schonend wie möglich anwenden, muss sie Massnahmen in verschiedensten Bereichen treffen.

\section{B4 Behandlung auf wissenschaftlicher Grundlage}

Die Qualität der Behandlung wird verbessert, wenn sie sich auf wissenschaftliche Evidenz abstützt. Der Standard fordert die Verantwortlichen auf, sich über den neuesten Stand des Wissens kontinuierlich zu informieren, das relevante Wissen in die klinische Praxis einzubauen und dafür zu sorgen, dass - wo notwendig und sinnvoll - auch nach dieser definierten Praxis verfahren wird. Neben international verfügbarem Basiswissen müssen regional unterschiedliche Gegebenheiten, Auffassungen und Gewohnheiten berücksichtigt werden.

\section{B5 Patientenzufriedenheit}

Der Begriff "Patientenzufriedenheit» ist letztlich zu eng gefasst. Der Standard schliesst andere Formen der Selbstbeurteilung und -bewertung mit ein, z.B. die Selbstbeurteilung des Gesundheits- bzw. Krankheitszustandes und/oder der Lebensqualität. Das Konzept Patientenzufriedenheit kennt die Medizin schon lange. Ein zufriedener Patient hält sich eher an die Behandlungsregeln und wird eher gesund. Er bewertet zudem den Behandlungserfolg positiver. In den letzten Jahren hat der Begriff "Patientenzufriedenheit» eine ganz neue Qualität erhalten. Der Patient begegnet der Klinik zunehmend nicht mehr nur als Patient, d.h. als leidender Mensch, der Heilung oder Linderung sucht. Er sieht sich immer mehr auch in der Rolle des Kunden, der eigene Bedürfnisse, Prioritäten und Forderungen hat.

\section{U1 Interdisziplinäre Zusammenarbeit}

In psychiatrischen Kliniken wird interdisziplinär gearbeitet. Daran entzünden sich im klinischen Alltag viele Konflikte und Schwierigkeiten. Eine Optimierung der Arbeit an der Nahtstelle zwischen den verschiedenen beteiligten Berufsgruppen ist wichtig. Das Qualitätsniveau der internen Zusammenarbeit wird nicht abstrakt, sondern in der konkreten Arbeit am und mit dem Patienten definiert. Der Standard fokussiert deshalb auf die interdisziplinäre Zusammenarbeit im Behandlungsprozess.

\section{U2 Umgang mit Patientendaten}

Der Titel des Standards heisst ganz bewusst «Umgang mit Patientendaten" und nicht etwa "Schutz der Patientendaten». Der Standard spricht damit ein Spannungsfeld an, in dem sich die Psychiatrischen Kliniken bewegen. Sie müssen einerseits Datenflüsse fördern, etwa um die Kontinuität der Behandlung oder die Kooperation der Angehörigen zu gewähr- 
leisten. Andererseits dürfen sie Daten nicht weitergeben, um die Persönlichkeitsrechte der Patienten nicht zu beeinträchtigen. Die Klinik befindet sich im Umgang mit Patientendaten auf einer Gratwanderung. In einem Prozess ständiger Interessenabwägung balanciert sie zwischen Schutz und Offenlegung, zwischen Patienten-, Dritt- und Eigeninteressen.

\section{U3 Das zielorientierte Mitarbeitergespräch}

Die Qualität der Behandlung kann nur so gut sein wie die Leistungen der Mitarbeitenden. Die Mitarbeitenden sind die wichtigste Ressource der Klinik. Der Umgang mit Mitarbeitenden hat sich zu einem eigenen Gebiet der Managementlehre entwickelt. Ein Kernelement und wichtiges Instrument des Human Ressource Managements ist das Mitarbeitergespräch. Fördernde Mitarbeitergespräche und Zielvereinbarungen haben positive Auswirkungen auf das Arbeitsklima, die Auftragserfüllung und damit letztlich auf die Behandlung.

\section{U4 Integriertes medizinisch-ökonomisches Denken und Handeln}

Das Umfeld der Kliniken hat sich in den letzten Jahren stark verändert. Die knapper werdenden finanziellen Ressourcen haben dazu geführt, dass die Wirtschaftlichkeit einer Behandlung immer wichtiger wird. Das Kriterium Wirtschaftlichkeit bzw. Kosteneffizienz ist zum Qualitätskriterium geworden. Der anhaltende Kosten- und Wettbewerbsdruck schafft günstige Voraussetzungen für eine Intensivierung und vor allem Verbesserung des integrierten medizinisch-ökonomischen Denkens und Handelns. Die verschiedenen Berufsgruppen müssen mehr als bisher die Standpunkte der anderen zur Kenntnis nehmen. Gesucht wird darüber hinaus eine neue Strategie, die Defensiv- und Abgrenzungshaltungen überwindet und gemeinsame Spielräume eröffnet. 